\title{
The HI distribution of spiral galaxies in the cluster A 262
}

\author{
H. Bravo-Alfaro ${ }^{1}$, A. Szomoru ${ }^{2,3}$, V. Cayatte $^{1}$, C. Balkowski ${ }^{1}$, and R. Sancisi ${ }^{2}$ \\ 1 Observatoire de Paris, DAEC and URA 173, associé au CNRS et à l'Université Paris 7, 92195 Meudon Cedex, France \\ 2 Kapteyn Astronomical Institute, P.O. Box 800, NL 97000 AV Groningen, The Netherlands \\ ${ }^{3}$ UCO/Lick Observatory, University of California, Santa Cruz, California 95064, U.S.A.
}

Received October 31, 1996; accepted April 21, 1997

\begin{abstract}
We present results of an Hi survey of the cluster of galaxies Abell 262, using the Westerbork Synthesis Radio Telescope (WSRT). Eleven galaxies were detected in five fields. In a few cases the gas in these galaxies shows an asymmetrical distribution; the most likely explanation is the interaction between the cluster medium and the gaseous disks. Only two of the detected galaxies are slightly Hi-deficient. At $40^{\prime \prime}$ to the West of CGCG $160-$ 049 we tentatively detect what may be an associated HI cloud. The total mass of this cloud is $2.510^{8} M_{\odot}$, and no optical counterpart has yet been found.
\end{abstract}

Key words: galaxies: clusters: individual: Abell 262 radio lines: galaxies

\section{Introduction}

This paper describes Hi observations of eleven galaxies in the cluster Abell 262. This is a spiral rich cluster, classed III in the Bautz-Morgan system and characterized by the $\mathrm{X}$-ray source $3 \mathrm{U} 015+36$, located near the core and concentric with respect to the elliptical galaxy NCG 708 . Its X-ray luminosity $\left(L_{\mathrm{x}}=3.0910^{43} \mathrm{ergs} \mathrm{s}^{-1}\right.$; Jones \& Forman 1984) is comparable to that of the Virgo cluster. Its relatively low redshift $(z=0.0156)$ combined with its wealth of spirals, makes it an interesting cluster for a study of the Hi content of its member galaxies as a parameter of environmental conditions.

The $21 \mathrm{~cm}$ line of neutral hydrogen is a very useful tool for probing the evolution of spirals in a cluster environment. Through comparisons of the Hi content of isolated galaxies with galaxies in Virgo (Chamaraux et al. 1980; Giovanelli \& Haynes 1983; Cayatte et al. 1990, 1994), and in other clusters (e.g. Giovanelli \& Haynes 1985) it has

Send offprint requests to: H. Bravo-Alfaro; Observatoire de Paris, F-92195 Meudon Cedex, France.

* Based on observations done at the Westerbork Synthesis Radio Telescope, in The Netherlands. been shown that cluster galaxies can be substantially deficient in neutral hydrogen. The Hi content of individual galaxies depends on the distance from the cluster center, with the most gas deficient objects near the core. Furthermore, the Hi deficiency of the cluster is also correlated with its $\mathrm{X}$-ray luminosity.

In order to obtain more information on the spatial distribution of the neutral hydrogen in deficient galaxies in A 262, $21 \mathrm{~cm}$ line observations were done with the WSRT. Five fields were surveyed, centered between 0.07 and 0.70 $r_{\mathrm{A}}$ (the Abell radius $r_{\mathrm{A}}$, equals $1.75^{\circ}$ for A 262), covering a significant part of the core of the cluster where we searched for galaxies already detected by Giovanelli et al. (1982), Giovanelli \& Haynes (1985) and Wegner et al. (1993).

We describe the observations and the data reduction in Sect. 2. Results are given in Sect. 3, where we present the total Hi maps of eleven galaxies superposed on optical images from the Digitized Sky Survey (DSS). For some of these galaxies (NGC 688 and NGC 753), we show the intensity weighted velocity fields, and position velocity diagrams along the major axis (for NGC 688, NGC 753 and UGC 1347). Our conclusions are summarized in Sect. 4.

\section{Observations and data reduction}

The observations were done in 1984 with the WSRT (Högbom \& Brouw 1974). Each pointing was observed for 12 hours, with a total bandwidth of $10 \mathrm{MHz}$ and 31 frequency channels, resulting in a channel spacing of $68 \mathrm{~km} \mathrm{~s}^{-1}$. The velocity resolution is 1.2 times the velocity spacing between adjacent channels. The calibration was done according to the standard WSRT procedures, described by van Someren-Greve (1974). The observational parameters are shown in Table 1.

For the observed fields the synthesized beam is about $13^{\prime \prime} \times 20^{\prime \prime}$. Our final sample consists of ten spirals and one irregular out of nearly 30 candidates. Only two of the detected galaxies (NGC 710 and CGCG $522-049$ ) are slightly HI deficient.

The WSRT data reduction program NEWSTAR was used to transform the $\mathrm{UV}$ data to $\left(\alpha, \delta, V_{\text {hel }}\right)$ cubes. 
Table 1. Observational parameters

\begin{tabular}{|c|c|c|c|c|c|c|c|c|c|}
\hline$\overline{(1)}$ & $\overline{(2)}$ & $\overline{(3)}$ & $\overline{(4)}$ & $\overline{(5)}$ & $\overline{(6)}$ & $\overline{(7)}$ & $\overline{(8)}$ & $7(9)$ & $7(10)$ \\
\hline Field & $\begin{array}{c}\alpha_{1950} \\
(\mathrm{~h} \mathrm{~m} \mathrm{~s})\end{array}$ & $\begin{array}{l}\delta 1950 \\
\circ / \prime \prime\end{array}$ & $\begin{array}{c}t_{\text {int }} \\
\text { (hrs.) }\end{array}$ & $\underset{\prime \prime}{\text { Beam size }}$ & $\begin{array}{l}\text { Bandwidth } \\
(\mathrm{MHz})\end{array}$ & $\begin{array}{c}\text { Central vel. } \\
\left(\mathrm{km} \mathrm{s}^{-1}\right)\end{array}$ & $\begin{array}{c}\Delta v \\
\left(\mathrm{~km} \mathrm{~s}^{-1}\right)\end{array}$ & $\begin{array}{c}\mathrm{rms} \\
\mathrm{mJy} / \text { beam }\end{array}$ & $\begin{array}{c}\mathrm{mJy}-\mathrm{K} \text { conv } \\
\mathrm{mJy} / \text { beam }\end{array}$ \\
\hline 1 & 14500.0 & 360000.0 & 12 & $12.4 \times 19.3$ & 10 & 4700 & 68.1 & 0.89 & 0.37 \\
\hline 2 & 14700.0 & $\begin{array}{lll}35 & 0500.0\end{array}$ & 12 & $15.2 \times 21.6$ & 10 & 4750 & 68.1 & 0.95 & 0.52 \\
\hline 3 & 14930.0 & 355000.0 & 12 & $15.6 \times 21.2$ & 10 & 5400 & 68.1 & 1.04 & 0.53 \\
\hline 4 & 15100.0 & 362500.0 & 12 & $11.7 \times 19.5$ & 10 & 5100 & 68.1 & 0.93 & 0.36 \\
\hline 5 & 15300.0 & 355000.0 & 12 & $13.7 \times 22.0$ & 10 & 5000 & 68.1 & 0.86 & 0.48 \\
\hline
\end{tabular}

Column 1: Field number.

Columns 2 and 3: R.A. and Dec. (1950) of each pointing.

Column 4: Integration time.

Column 5: Minor and major axes of the synthesized beams, in seconds of arc.

Column 6: Total observed bandwidth in $\mathrm{MHz}$.

Column 7: Heliocentric velocity of the central channel.

Column 8: Channel separation in $\mathrm{km} \mathrm{s}^{-1}$. The velocity resolution is equivalent to 1.2 times this velocity spacing.

Column 9: rms noise per channel after continuum subtraction, in $\mathrm{mJy} / \mathrm{beam}$.

Column 10: The flux density per beam area (in $\mathrm{mJy} /$ beam) equivalent to $1.0 \mathrm{~K}$ in the channel maps.

Table 2. Observed galaxies

\begin{tabular}{llllccrrrr}
\hline \hline$(1)$ & $(2)$ & $(3)$ & $(4)$ & $(5)$ & $(6)$ & $(7)$ & $(8)$ & $(9)$ & 10 \\
Name & $\alpha_{1950}$ & $\delta_{1950}$ & Type & mag & $a \times b$ & $i$ & PA & $r / r_{\mathrm{A}}$ & Hi Def \\
\hline (1) NGC 668 & 14327.2 & 361240 & Sb & 12.64 & $1.8 \times 1.2$ & $50^{1}$ & $33^{1}$ & 0.77 & 0.03 \\
(2) UGC 1257 & 14511.3 & 361213 & Sab & 14.59 & $1.0 \times 0.5$ & $64^{2}$ & $107^{2}$ & 0.57 & 0.11 \\
(3) UGC 1277 & 14630.7 & 351214 & S0a & 14.03 & $1.7 \times 0.9$ & $62^{2}$ & $75^{2}$ & 0.55 & 0.22 \\
(4) UGC 1299 & 14737.6 & 350639 & Im & 15.69 & $1.0 \times 0.6$ & $65^{2}$ & $85^{2}$ & 0.53 & -0.17 \\
(5) NGC 688 & 14748.8 & 350213 & SBb(rs)p & 12.43 & $2.4 \times 1.5$ & $55^{1}$ & $146^{1}$ & 0.56 & 0.10 \\
(6) NGC 710 & 14957.5 & 354826 & Scd & 13.79 & $1.7 \times 1.7$ & $19^{2}$ & $51^{4}$ & 0.06 & 0.38 \\
(7) UGC 1347 & 14949.0 & 362221 & SABc(rs) & 13.23 & $1.3 \times 1.1$ & $30^{1}$ & $18^{1}$ & 0.26 & -0.07 \\
(8) CGCG 522-049 & 15053.7 & 361907 & SBd $^{1}$ & 15.10 & $0.9 \times 0.7$ & $45^{2}$ & $149^{3}$ & 0.26 & 0.33 \\
(9) UGC 1361 & $15056.2^{1}$ & $362027^{1}$ & Scd & 15.43 & $1.1 \times 0.4$ & $73^{2}$ & $131^{3}$ & 0.27 & 0.12 \\
(10) UGC 1387 & 15213.8 & 360100 & Sdm & 15.37 & $0.9 \times 0.6$ & $48^{2}$ & $175^{2}$ & 0.27 & -0.06 \\
(11) NGC 753 & 15445.4 & 354022 & SABbc(rs) & 11.66 & $2.5 \times 2.0$ & $50^{1}$ & $128^{1}$ & 0.58 & -0.15 \\
\hline
\end{tabular}

Column 1: Galaxy identification.

Columns 2 and 3: 1950 R.A. and Dec. from RC3 except for (1), taken from Paturel et al. (1989).

Column 4: Morphological type from RC3 except for (1), taken from the LEDA data base.

Column 5: Blue magnitude after corrections for redshift, galactic and internal extinction (Wegner et al. 1993).

Column 6: Optical major and minor axis in minutes of arc, from the UGC when available, else from Wegner et al. (1993).

Column 7: Inclination angle; from Amram et al. (1994) (1), and the LEDA base data (2).

Column 8: Position angle from Amram et al. (1994) (1), Nilson (1973) (2), Gavazzi et al. (1995) (3), and Scodeggio et al. (1992) (4).

Column 9: Distance from the cluster center in units of Abell radii $\left(r_{\mathrm{A}}=1.75^{\circ}\right.$ for A 262).

Column 10: The Hi deficiency is defined as the difference between the observed Hi mass and that expected for an isolated galaxy of the same optical linear diameter and morphological type (Giovanelli \& Haynes 1985).

Further data reduction was done with the Groningen Image Processing System (GIPSY). First a continuum map was constructed by averaging channels at the high and low end of the passband; first and last channels were always excluded because of their higher noise levels. This continuum map was then subtracted and the channel maps were inspected for $21 \mathrm{~cm}$ line emission. A new continuum map was made for each detected galaxy using only line-free channels, and it was subtracted from the original cube. After the continuum subtraction, maps were CLEANed and restored with a Gaussian beam with the same FWHM as the original beam, in order to remove instrumental effects. The CLEAN procedure was done to a depth of $0.5 \sigma$, the rms noise in the cubes. The maps were then smoothed spatially, in order to improve the signal-tonoise ratio of extended emission. The beam size typically used was $30^{\prime \prime} \times 30^{\prime \prime}$, except for NGC 710 , where a $45^{\prime \prime} \times 45^{\prime \prime}$ beam was used. In order to make the total Hi maps, velocity fields and position-velocity plots, shown in the next section, line emission was isolated using the mask (or conditional transfer) procedure. The main parameters of the eleven detected galaxies are listed in Table 2. 
Table 3. Hi parameters of the detected galaxies

\begin{tabular}{|c|c|c|c|c|}
\hline$\overline{(1)}$ & $\overline{(2)}$ & $(3)$ & $(4)$ & $\overline{(5)}$ \\
\hline Name & $\begin{array}{l}v_{\text {hel }} \\
\mathrm{km} \mathrm{s}^{-1}\end{array}$ & $\begin{array}{l}W_{20} \\
\mathrm{~km} \mathrm{~s}^{-1}\end{array}$ & $\begin{array}{r}\text { Hi flux } \\
\text { Jy } \mathrm{km} \mathrm{s}^{-1}\end{array}$ & $\begin{array}{r}F_{\text {cont }} \\
(\mathrm{mJy})\end{array}$ \\
\hline (1) NGC 668 & 4533 & 318 & $5.73 \pm 0.37$ & $15.9 \pm 0.3$ \\
\hline (2) UGC 1257 & 4667 & 417 & $2.46 \pm 0.14$ & $4.2 \pm 0.4$ \\
\hline (3) UGC 1277 & 4128 & 617 & $3.02 \pm 0.31$ & $4.8 \pm 0.4$ \\
\hline (4) UGC 1299 & 5498 & 235 & $3.23 \pm 0.25$ & $1.3 \pm 0.3$ \\
\hline (5) NGC 688 & 4166 & 455 & $7.50 \pm 0.39$ & $12.7 \pm 0.4$ \\
\hline (6) NGC 710 & 6111 & 316 & $2.16 \pm 0.19$ & $24.4 \pm 0.5$ \\
\hline (7) UGC 1347 & 5551 & 183 & $4.46 \pm 0.27$ & $14.9 \pm 0.5$ \\
\hline (8) CGCG 522-049 & 4691 & 244 & $1.32 \pm 0.08$ & $1.6 \pm 0.4$ \\
\hline (9) UGC 1361 & 5749 & 291 & $1.89 \pm 0.15$ & $1.4 \pm 0.3$ \\
\hline (10) UGC 1387 & 4548 & 265 & $2.46 \pm 0.21$ & $1.1 \pm 0.4$ \\
\hline (11) NGC 753 & 4890 & 468 & $18.03 \pm 1.04$ & $19.0 \pm 0.2$ \\
\hline
\end{tabular}

Column 1: Galaxy name.

Column 2: Heliocentric velocity, derived from the HI profile. The uncertainty is of the order of half the velocity resolution, i.e. $41 \mathrm{~km} \mathrm{~s}^{-1}$. Column 3: $21 \mathrm{~cm}$ profile width, at a level of $20 \%$ of the peak value. Uncertainty in the widths are of the order of the velocity resolution.

Column 4: $21 \mathrm{~cm}$ total line flux and the corresponding error.

Column 5: $21 \mathrm{~cm}$ continuum flux. Uncertainty is equal to $1 \sigma$ in the continuum map.

Table 4. Parameters of the undetected galaxies

\begin{tabular}{lccrrrrr}
\hline \hline$(1)$ & $(2)$ & $(3)$ & $(4)$ & $(5)$ & $(6)$ & $(7)$ & $(8)$ \\
Name & field & $\alpha_{1950}$ & $\delta_{1950}$ & $v_{\text {hel }}$ & $W_{20}$ & Hi flux & source \\
\hline CGCG 522-024 & 3 & 14833.1 & 354906 & 5311 & 258 & 1.56 & $(1)$ \\
CGCG 522-053 & 4 & 15059.9 & 362342 & 4556 & - & $<1.15$ & $(2)$ \\
CGCG 522-055 & 4 & 15122.6 & 362307 & 5121 & 405 & 0.93 & $(1)$ \\
CGCG 522-071 & 5 & 15307.8 & 355308 & 5336 & 962 & 1.37 & $(1)$ \\
\hline \hline
\end{tabular}

Column 1: Galaxy name.

Column 2: Field where detection was expected.

Columns 3 and 4: 1950 R.A. and Dec.

Column 5: Heliocentric velocity, in $\mathrm{km} \mathrm{s}^{-1}$.

Column 6: Observed $21 \mathrm{~cm}$ profile width, in $\mathrm{km} \mathrm{s}^{-1}$, measured at a level of $20 \%$ of the mean signal intensity.

Column 7: The $21 \mathrm{~cm}$ line flux in Jy km s${ }^{-1}$.

Column 8: References for Hi data. (1) Wegner et al. (1993), (2) Scodeggio \& Gavazzi (1993).

\section{Results}

The Hi parameters of the observed galaxies, obtained from our WSRT observations, are given in Table 3. The Hi flux values are corrected for the WSRT primary beam attenuation. The total hydrogen content and heliocentric velocities agree closely with previous results obtained from single dish observations (e.g. Giovanelli et al. 1982, Giovanelli \& Haynes 1985, and Wegner et al. 1993). This shows that the synthesis observations did not miss a significant fraction of HI, except for NGC 668 and NGC 753, which are located far from their corresponding pointing centers. Due to the primary beam response of the WSRT, roughly a Gaussian with a FWHM of $38^{\prime}$, the noise in the maps increases with radius causing a corresponding loss of signalto-noise. Finally, detections far from the pointing centers have larger uncertainties in their fluxes. The velocity widths reported in Table 4 are systematically larger, within our uncertainty, than those previously published by Giovanelli Haynes \& Haynes (1982), and Wegner et al. (1993). The cause of this discrepancy is probably the low signal-to-noise ratio combined with the uncertainty in our velocity widths, which is of the order of the resolution $\left(80 \mathrm{~km} \mathrm{~s}^{-1}\right)$.

In Figs. 1-4, 5a, 6, 7a, 8, 9, and 10a we show the HI maps for each galaxy, with Hi contours superposed on optical images from the DSS. Figures $5 \mathrm{~b}$ and $10 \mathrm{~b}$ show velocity fields for the largest objects in our sample, NGC 688 and NGC 753. For three galaxies (NGC 688, UGC 1347, and NGC 753) we have also plotted the position-velocity diagram together with the $\mathrm{H} \alpha$ rotation curves obtained by Amram et al. 1994 (Figs. 5c, 7b, and 10c). Position angles are also taken from that work. The central velocities used in these plots were taken from Wegner et al. (1993). 
Table 4 lists four undetected galaxies located in our observed fields, with total flux or upper limit higher than $0.9 \mathrm{Jy} \mathrm{km} \mathrm{s}^{-1}$, as measured from single dish observations. The first, CGCG 522-024, remained undetected because its large distance from the pointing center. In adittion, the field where we expected this galaxy has the highest noise among the five observed fields (see Table 1). CGCG 522 - 071 was not detected because its Hi emission is spread out over a width of almost $1000 \mathrm{~km} / \mathrm{s}$ and is consequently very diluted. CGCG $522-055$ has a total flux of 0.93 , which is lower than our detection threshold. Finally, CGCG 522 - 053 has not been detected in previous single dish $21 \mathrm{~cm}$ line surveys.

In the following we briefly discuss each detected galaxy.

- NGC 668 is a bright spiral (Sb) located at a relatively large projected distance from the cluster center $\left(81^{\prime}\right)$. The signal-to-noise ratio of this detection is low because of its large distance (nearly $22^{\prime}$ ) from the pointing center. The first contour in Fig. 1 is $4.710^{20} \mathrm{~cm}^{-2}$. More sensitive measurement are needed to confirm the observed irregular distribution.

- UGC 1257, UGC 1277 and UGC 1299. These galaxies are located at roughly half the Abell radius from the cluster center (see Table 2), and they have a fairly normal neutral hydrogen content. The HI distributions, shown in Figs. 2 to 4, extend beyond the optical disks, and no evidence of interaction with neighboring objects or the intracluster medium is seen. Only in the case of UGC 1257 a slight misalignment between the optical and radio major axis is visible.

- NGC 688. The Hi map of this galaxy (Fig. 5a) shows a slight asymmetry in the NW. This could be an extension of the northern optical arm which is pointing towards the neighboring irregular galaxy UGC 1299 . Correspondingly, the velocity field (Fig. 5b) also shows a disrupted contour in the same region. However, these galaxies are rather far apart to be interacting: $4.5^{\prime}$ and $1300 \mathrm{~km} \mathrm{~s}^{-1}$. In Fig. $5 \mathrm{c}$ we show the positionvelocity diagram and the $\mathrm{H} \alpha$ rotation curve obtained by Amram et al. (1994), who explain this rotation curve as due to the presence of a central bar. Even if the spatial and velocity resolution of the $\mathrm{HI}$ data are not sufficient to confirm the increase seen at the end of the $\mathrm{H} \alpha$ rotation curve (Amram et al. 1994), we can at least exclude a decreasing feature.

- NGC 710. This is the only detected galaxy in the field pointed towards the cluster center, and one of the two deficient galaxies in our sample (Def $=0.38$, Giovanelli \& Haynes 1985). Our flux of $2.16 \mathrm{Jy} \mathrm{km} \mathrm{s}^{-1}$ is slightly lower than the one obtained by Wegner et al. (1993) (2.51 $\mathrm{Jy} \mathrm{km} \mathrm{s}^{-1}$ ). This difference could be explained because NGC 710 falls at the edge of the velocity band. A stronger smoothing was necessary to detect this galaxy, leading to a lower resolution than the one used for the other objects. Therefore, the asymmetry seen in the HI map, which arises from signal in only one channel, should be taken with caution. This asymmetry coincides with an optical counterpart with unknown velocity. The Hi emission, seen in five channels, indicates that the dynamical axis lies in the East-West direction, contrary to the optical major axis $\left(\mathrm{PA}=51^{\circ}\right)$. The receding side being in the West part. NGC 710 is seen on projection on the X-ray source, and its velocity is higher by $\sim 1300 \mathrm{~km} \mathrm{~s}^{-1}$ compared to the mean cluster velocity making this galaxy a good candidate to show environmental effects.

- UGC 1347. The map in Fig. 7a suggests that the HI gas extend well beyond the optical disk, with a slight excess on the West side. The neutral hydrogen data and the $\mathrm{H} \alpha$ rotation curve (Amram et al. 1994) are in good agreement (Fig. 7b). Our Hi data confirm the flatness of the $\mathrm{H} \alpha$ rotation curve even beyond $r_{25}$, the corrected de Vaucouleurs radius at the 25th magnitude $\left(r_{25}=39.5^{\prime \prime}\right.$ for this galaxy).

- UGC 1361 and CGCG 522 - 049 are shown in Fig. 8. These galaxies are projected close to the cluster center. UGC 1361 has a normal Hi content, with an hydrogen distribution which is much more extended than the optical disk. One remarkable feature is the clear asymmetry in the NW region, where the maximum of the $\mathrm{HI}$ emission is seen without an optical counterpart. CGCG 522-049 shows an asymmetric Hi distribution, with an extension on the opposite side of UGC 1361. In spite of the irregular hydrogen distributions and the small angular separation of only $1.5^{\prime}$, the evidence for interaction between these two galaxies is not conclusive; their velocity difference is more than $1000 \mathrm{~km} \mathrm{~s}^{-1}$. An interesting result, still to be confirmed, is the weak HI emission found towards the West of CGCG $522-$ 049. Inspection of the DDS did not reveal any obvious optical counterpart. The total HI mass is $2.410^{8} M_{\odot}$, typical of dwarf galaxies. This would imply a $M_{\mathrm{HI}} / L_{\mathrm{B}}$ ratio of at least 4.7, taking 19.5 as the magnitude limit in the DSS and a distance to A 262 of $47 \mathrm{Mpc}$. Such objects, commonly associated with larger galaxies, have already been noticed in some clusters: NGC 4694 in Virgo (Cayatte et al. 1990), CGCG 160 - 106 in Coma (Amram et al. 1992; Bravo-Alfaro et al. 1997), and several examples in Hydra (McMahon 1993). Tentative explanations for the Hi cloud found near NGC 4694, accretion or ejection from the larger galaxy nearby, have been proposed by van Driel \& van Woerden (1989).

- UGC 1387 is a late type galaxy (Sdm), low surface brightness spiral (Schneider et al. 1992). It is relatively 
close in projection to the cluster center, but it is not HI-deficient. The peak of Hi emission is offset from the optical center by about $15^{\prime \prime}$ towards the NW (Fig. 9).

- NGC 753 is one of the brightest spirals in the whole cluster, and the only one in our sample on which additional WSRT data are available (Broeils et al. 1994). In Fig. 10a two HI features can be identified in the southern region. They probably correspond to faint extensions of two spiral arms. Furthermore, these two structures point away from the direction of the cluster center. This asymmetric gas distribution, also evident in the velocity field of Fig. 10b, is in a region where the $\mathrm{H} \alpha$ map, from Amram et al. (1994), shows very few HII regions, while most of the brightest ones are in the northern part. Our position-velocity diagram confirms the overall shape of the $\mathrm{H} \alpha$ rotation curve (Fig. 10c). Our total flux measure is almost $50 \%$ lower than previous values (e.g. $31.4 \mathrm{Jy} \mathrm{km} \mathrm{s}^{-1}$ from Giovanelli \& Haynes 1982) maybe because of strong primary beam attenuation. This galaxy was observed at 22 minutes of arc from the pointing center.

\section{Concluding remarks}

We have obtained Hi observations of five fields in the cluster A 262 using the WSRT. Eleven galaxies, ten spirals and one irregular, were detected. The main result of our study is summarized in Fig. 11 where we show the total emission of the detected galaxies, located at their proper positions in the cluster, magnified in size by a factor of six. First contours are between 1.8 and $3.910^{20} \mathrm{~cm}^{-2}$, except for NGC $668\left(4.710^{20} \mathrm{~cm}^{-2}\right)$ and NGC $753\left(6.410^{20} \mathrm{~cm}^{-2}\right)$. We did not detect any of the most deficient galaxies in the core of the cluster.

Within a radius of one degree from the cluster center $(0.8 \mathrm{Mpc}$, assuming a distance to A 262 of $47 \mathrm{Mpc})$ we found galaxies to be more extended in $\mathrm{HI}$ than in the optical, except for NGC 710, located at the very center of the cluster. This is in contrast with the results obtained by Cayatte et al. (1990), who found galaxies in Virgo within $3^{\circ}$ from M 87 (0.9 Mpc, considering Virgo at a distance of $17.5 \mathrm{Mpc})$ to be severely stripped of their neutral hydrogen.

The three galaxies in the North East field (UGC 1347, CGCG 522 - 049, and UGC 1361), show Hi distributions slightly offset to the West of the optical position. These objects are close to the $\mathrm{X}$-ray source located at the center of the cluster. Other galaxies, NGC 688 and NGC 753, show also asymmetrical gas distributions. The cause of these effects could be ram-pressure exerted by the intracluster medium. A possible $\mathrm{HI}$ cloud was found near CGCG 522 - 049. If real, the presence of such an object in a cluster would be quite surprising as it would have to survive in an extremely hostile environment. Further HI mapping in the central regions of A 262, to be done with the VLA or the upgraded WSRT, would be useful to confirm the presence of this kind of objects as well as to explain the irregular gas distributions observed in this work.

Acknowledgements. We have made use of the Lyon-Meudon Extragalactic Database (LEDA) supplied by the LEDA team at the CRAL-Observatoire de Lyon (France). V.C. and C.B. acknowledge financial support from the Groupement de Recherche Cosmologie. H.B.A. thanks the Consejo Nacional de Ciencia y Tecnología, for its support through a Ph.D. Grant. The Westerbork Synthesis Radio Telescope is operated by the Netherlands Foundation for Research in Astronomy (NFRA/ASTRON), with financial support by the Netherlands Organization for Scientific Research (N.W.O.). We thank the referee of this article, G. Gavazzi, for his helpful comments.

\section{References}

Amram P., Le Coarer E., Marcelin M., et al., 1992, A\&AS 94, 175

Amram P., Marcelin M., Balkowski C., et al., 1994, A\&AS 103, 5

Bravo-Alfaro H., Cayatte V., van Gorkom J.H., Balkowski C., 1997 (in preparation)

Broeils A.H., van Woerden H., 1994, A\&AS 107, 129

Cayatte V., van Gorkom J.H., Balkowski C., Kotanyi C., 1990, AJ 100,604

Cayatte V., Kotanyi C., Balkowski C., van Gorkom J.H., 1994, AJ 107, 1003

Chamaraux P., Balkowski C., Gérard E., 1980, A\&A 83, 38

Gavazzi G., Boselli A., Carrasco L., 1995, A\&AS 112, 257

Giovanelli R., Haynes M.P., Chincarini G.L., 1982, ApJ 262, 442

Giovanelli R., Haynes M.P., 1983, AJ 88, 881

Giovanelli R., Haynes M.P., 1985, ApJ 292, 404

Högbom J.A., Brouw W.N., 1974, A\&A 33, 289

Jones C., Forman W., 1984, ApJ 276, 38

McMahon P.M., 1993, Ph.D. thesis, Columbia University, U.S.A.

Nilson P., 1973, Uppsala General Catalogue of Galaxies, Uppsala Astr. Obs. Annaler

Paturel G., Fouqué P., Bottinelli L., Gouguenheim L., 1989, Catalogue of Principal Galaxies. Observatoires de Lyon et Paris-Meudon

Schneider S.E., Thuan T.X., Mangum J.G., Miller J., 1992, ApJ 81, 5

Scodeggio M., Boselli A., Gavazzi G., Trinchieri G., Garilli B., 1992, A\&AS 94, 299

Scodeggio M., Gavazzi G., 1993, ApJ 409, 110

van Someren Greve H.W., 1974, A\&AS 15, 343

van Driel W., van Woerden H., 1989, A\&A 225, 317

Wegner G., Haynes M.P., Giovanelli R., 1993, AJ 105, 1251 


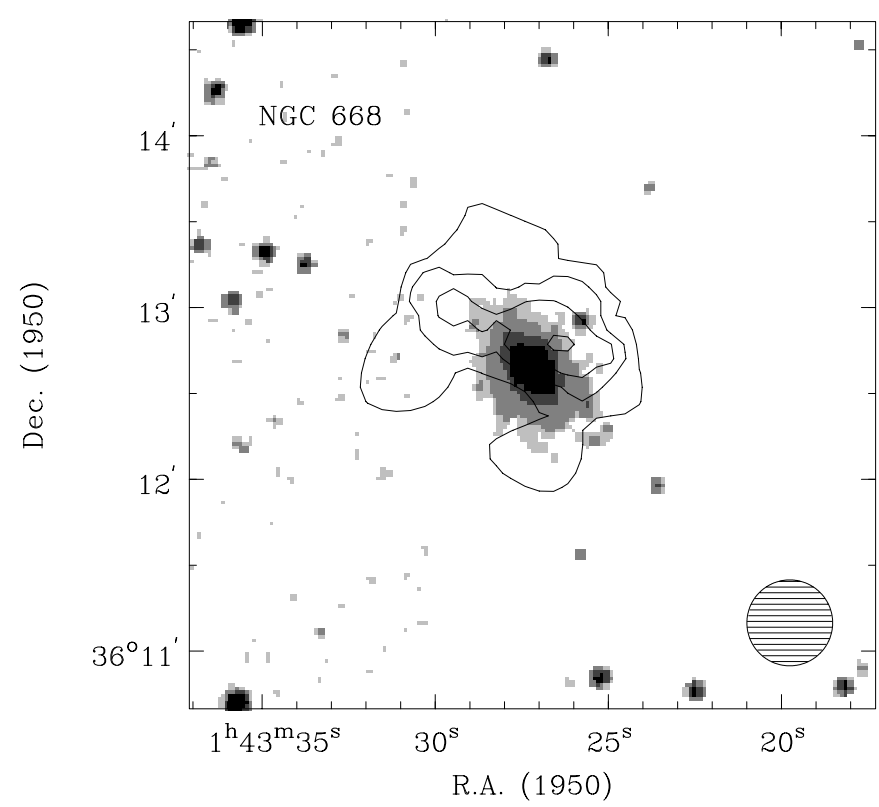

Fig. 1. Hi density distribution of NGC 668, superposed on a $B$-band gray scale image (from the DSS Survey). The contours are $4.7(2.0 \sigma), 9.4,14.1$, and $18.810^{20} \mathrm{~cm}^{-2}$. The FWHM is indicated by the hatched circle, $30^{\prime \prime} \times 30^{\prime \prime}$

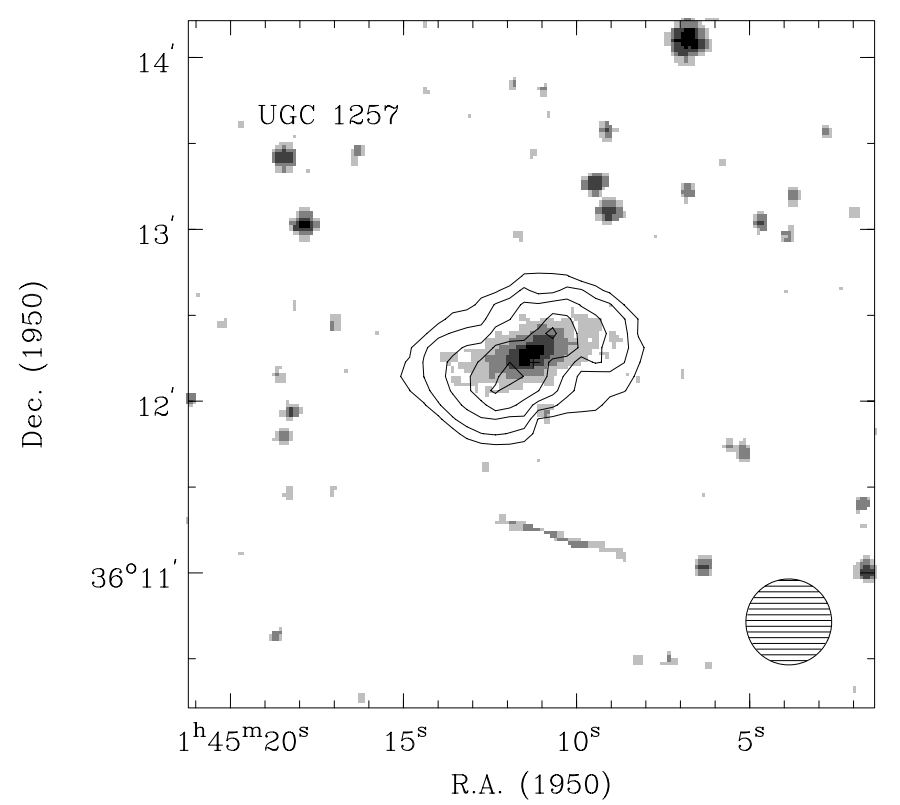

Fig. 2. HI density distribution of UGC 1257, superposed on a $B$-band gray scale image (from the DSS Survey). The contours are $2.9(2.5 \sigma), 5.8$ and $8.7,11.5$, and $14.410^{20} \mathrm{~cm}^{-2}$. The FWHM is indicated by the hatched circle, $30^{\prime \prime} \times 30^{\prime \prime}$

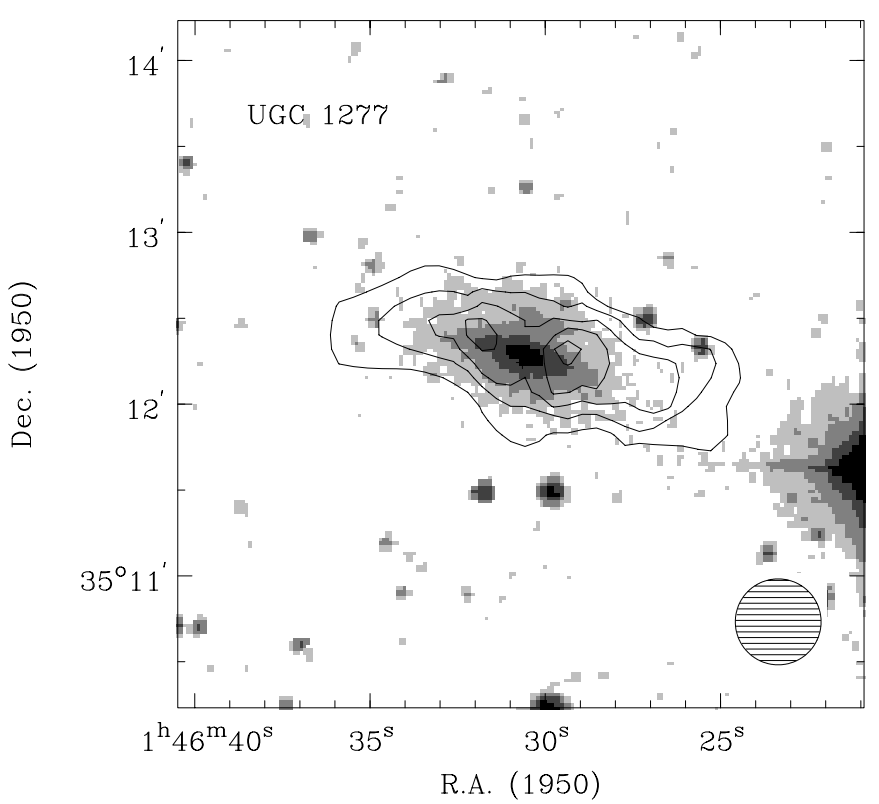

Fig. 3. HI density distribution of UGC 1277, superposed on a $B$-band gray scale image (from the DSS Survey). The contours are $2.3(2.5 \sigma), 4.7,7.0,9.4$, and $11.710^{20} \mathrm{~cm}^{-2}$. The FWHM is indicated by the hatched circle, $30^{\prime \prime} \times 30^{\prime \prime}$

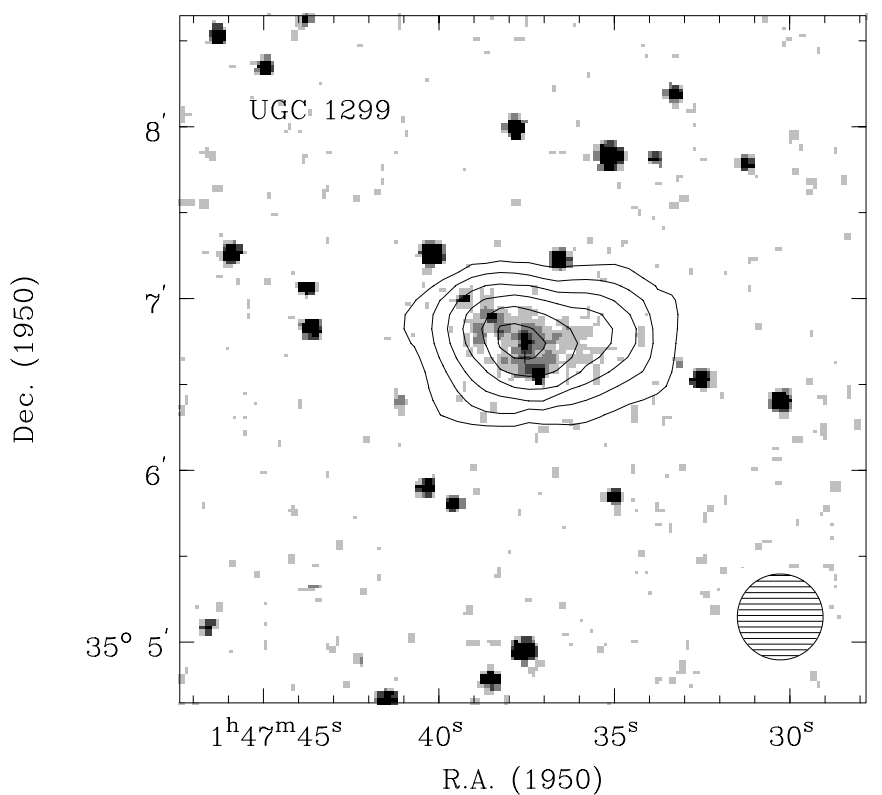

Fig. 4. HI density distribution of UGC 1299, superposed on a $B$-band gray scale image (from the DSS Survey). The contours are $2.7(2.5 \sigma), 5.4,8.1,10.8,13.5$, and $16.210^{20} \mathrm{~cm}^{-2}$. The FWHM is indicated by the hatched circle, $30^{\prime \prime} \times 30^{\prime \prime}$ 


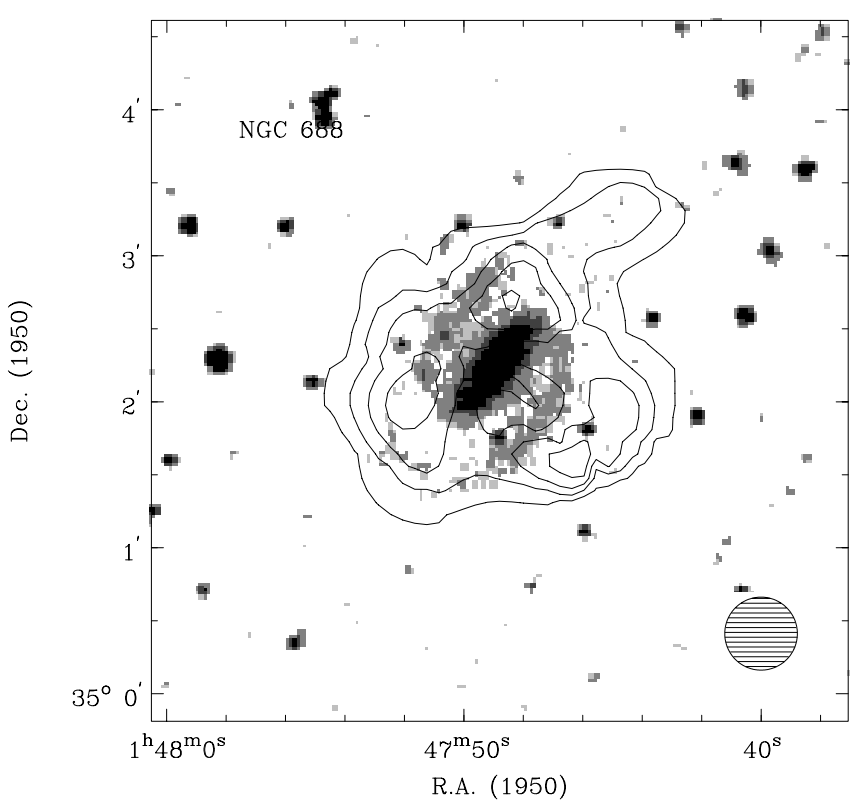

Fig. 5. a) HI density distribution of NGC 688 , superposed on a $B$-band gray scale image (from the DSS Survey). The contours are $2.4(2.5 \sigma), 4.8,7.3,9.7$, and $12.110^{20} \mathrm{~cm}^{-2}$. The FWHM is indicated by the hatched circle, $30^{\prime \prime} \times 30^{\prime \prime}$

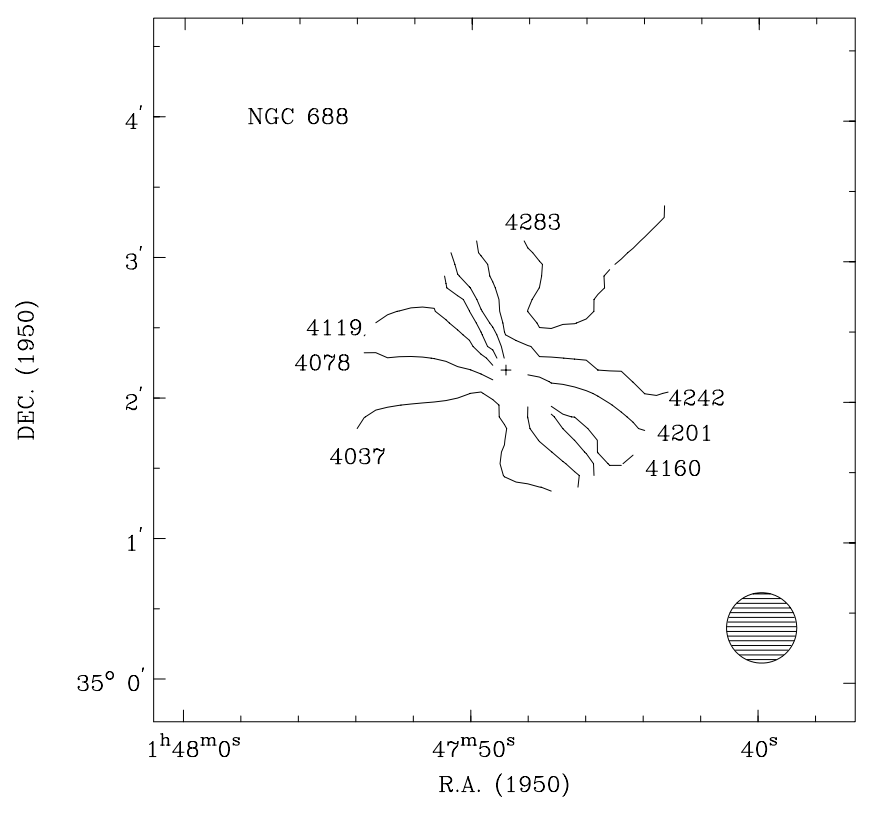

Fig. 5. b) Intensity weighted mean velocity field of NGC 688 . The numbers indicate heliocentric velocity in $\mathrm{km} \mathrm{s}^{-1}$. The FWHM is indicated by the hatched circle, $30^{\prime \prime} \times 30^{\prime \prime}$

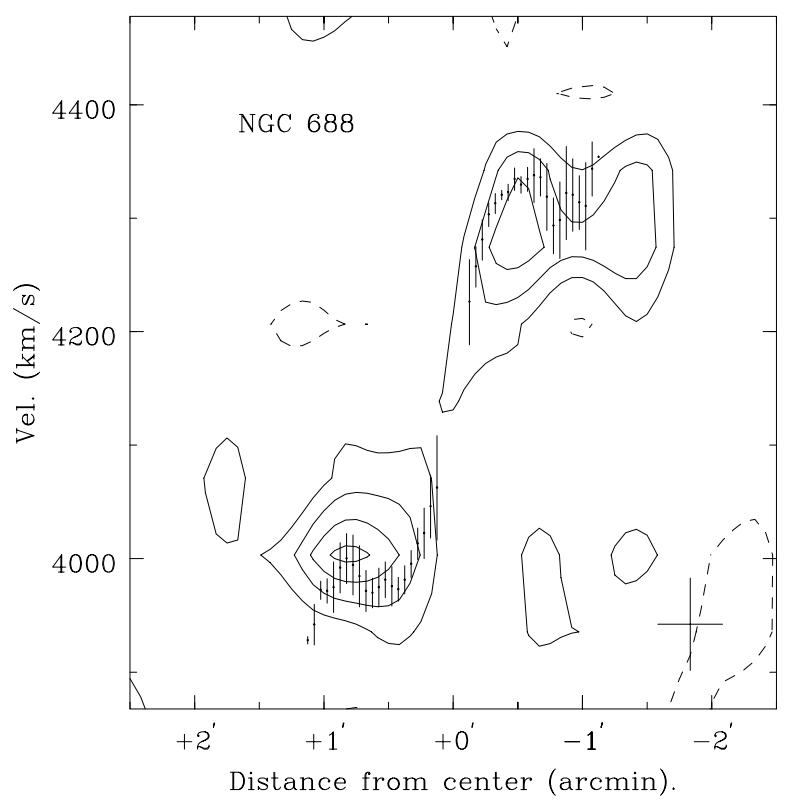

Fig. 5. c) Position velocity plot of NGC 688 along the major axis. The position angle is $146^{\circ}$. Contours are -1.3 (dashed lines), 1.3 to 5.2 mJy beam $^{-1}$ in steps of $1.3 \mathrm{mJy}$ beam $^{-1}$ area. The resolution is indicated by the cross in the lower right corner, the $\mathrm{H} \alpha$ rotation curve, from Amram et al. (1994), is plotted with the corresponding error bars

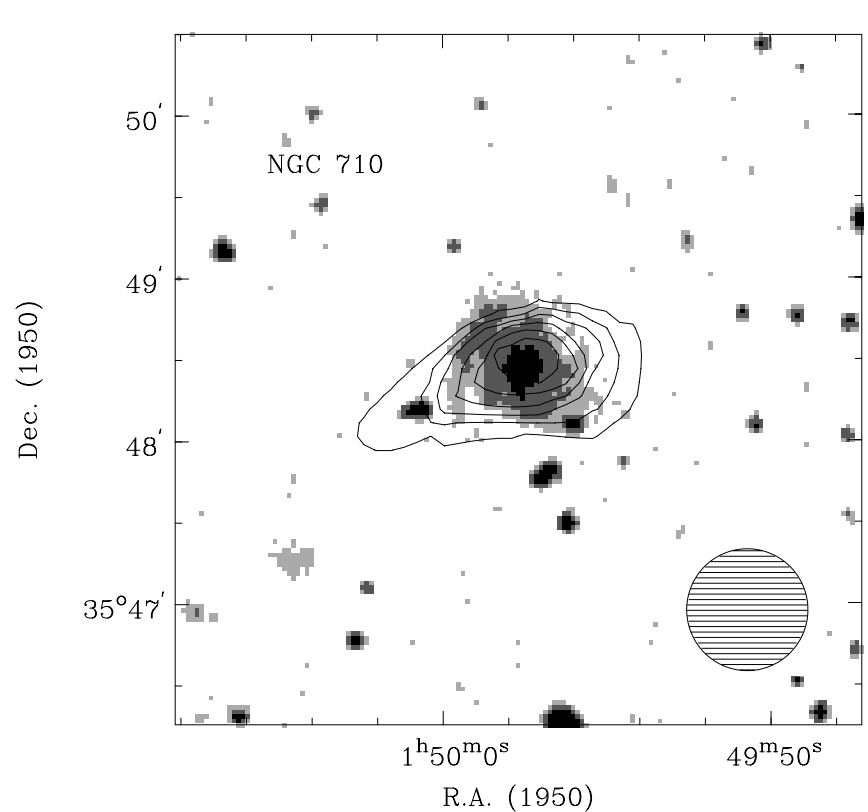

Fig. 6. HI density distribution of NGC 710, superposed on a $B$-band gray scale image (from the DSS Survey). The contours are $1.8(2.5 \sigma), 4.1,6.3,8.5,10.8,13.0$, and $15.210^{20} \mathrm{~cm}^{-2}$. The FWHM is indicated by the hatched circle, $45^{\prime \prime} \times 45^{\prime \prime}$ 


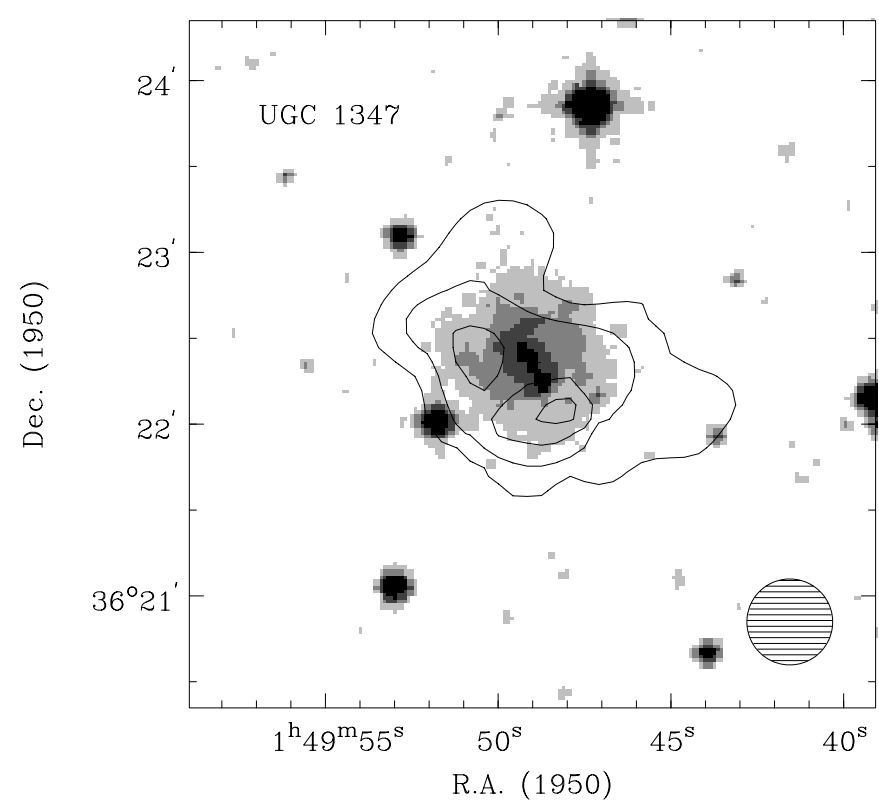

Fig. 7. a) HI density distribution of UGC 1347, superposed on a $B$-band gray scale image (from the DSS Survey). The contours are 3.2 (2.5 sigma), 6.5, 9.7 and $13.010^{20} \mathrm{~cm}^{-2}$. The FWHM is indicated by the hatched circle, $30^{\prime \prime} \times 30^{\prime \prime}$

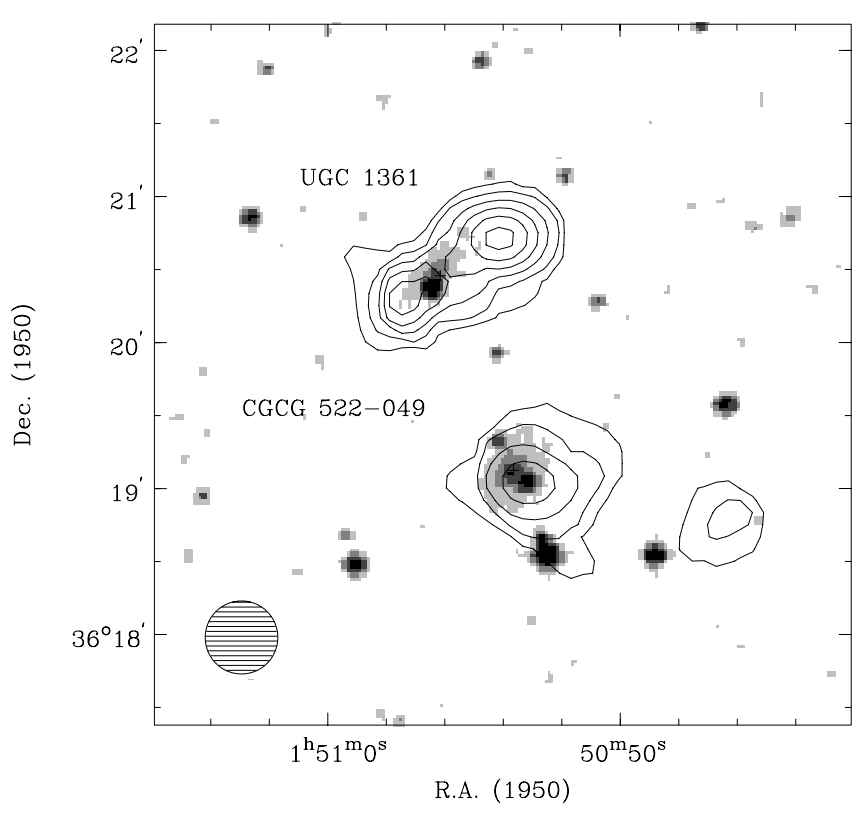

Fig. 8. HI density distribution of the couple UGC 1361 and CGCG 522 - 49, superposed on a $B$-band gray scale image (from the DSS Survey). For UGC 1361, the contours are $2.2(2.0 \sigma), 3.8,5.4,7.5,8.6$ and $10.210^{20} \mathrm{~cm}^{-2}$. For CGCG 522 - 49 and the third object in this figure, the contours are $2.2(2.5 \sigma), 3.8,5.4$ and $7.510^{20} \mathrm{~cm}^{-2}$. The FWHM is $30^{\prime \prime} \times 30^{\prime \prime}$

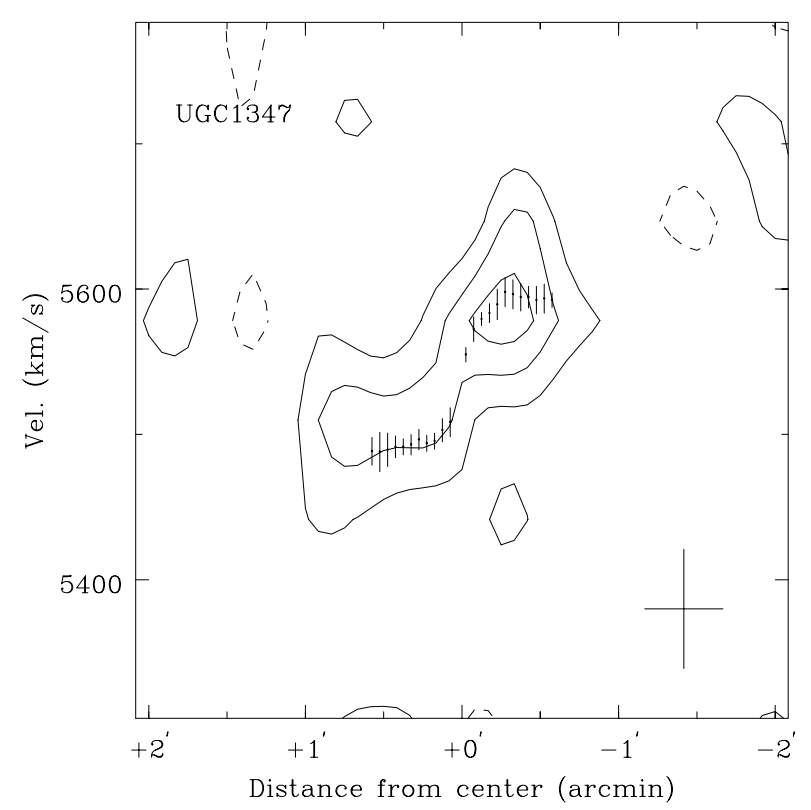

Fig. 7. b) Position velocity plot along the major axis, for UGC 1347. The position angle is $18^{\circ}$. Contours are -1.6 (dashed lines), 1.6 to $4.8 \mathrm{mJy}^{-1}$ beam $^{-1}$ in steps of $1.6 \mathrm{mJy}$ beam $^{-1}$. The resolution is indicated by the cross in the lower right corner, the $\mathrm{H} \alpha$ rotation curve, from Amram et al. (1994), is plotted with the corresponding error bars

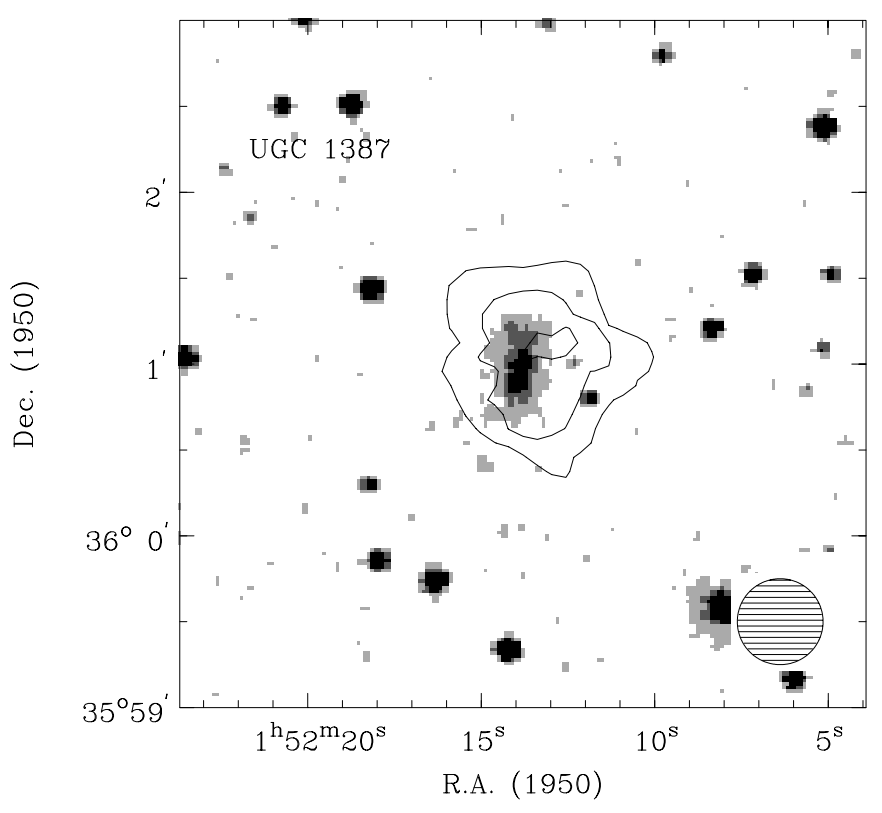

Fig. 9. HI density distribution of UGC 1387, superposed on a $B$-band gray scale image (from the DSS Survey). The contours are $3.9(2.5 \sigma), 7.7$, and $11.610^{20} \mathrm{~cm}^{-2}$. The FWHM is indicated by the hatched circle, $30^{\prime \prime} \times 30^{\prime \prime}$ 


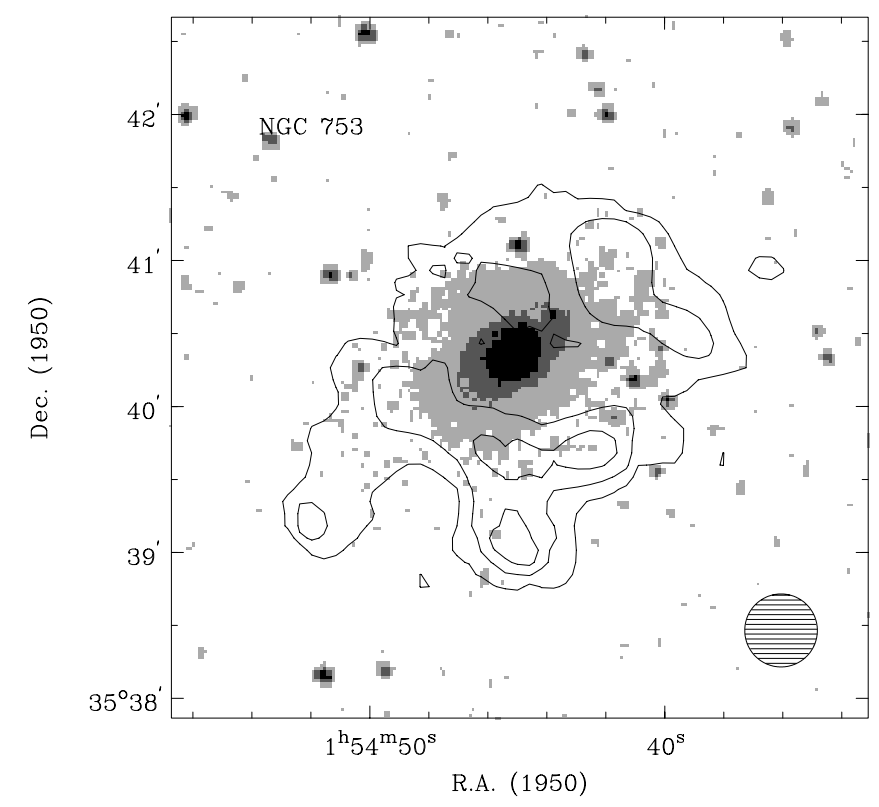

Fig. 10. a) HI density distribution of NGC 753, superposed on a $B$-band gray scale image (from the DSS Survey). The contours are $6.4(2.5 \sigma), 12.8$ and $19.210^{20} \mathrm{~cm}^{-2}$. The FWHM is indicated by the hatched circle, $30^{\prime \prime} \times 30^{\prime \prime}$

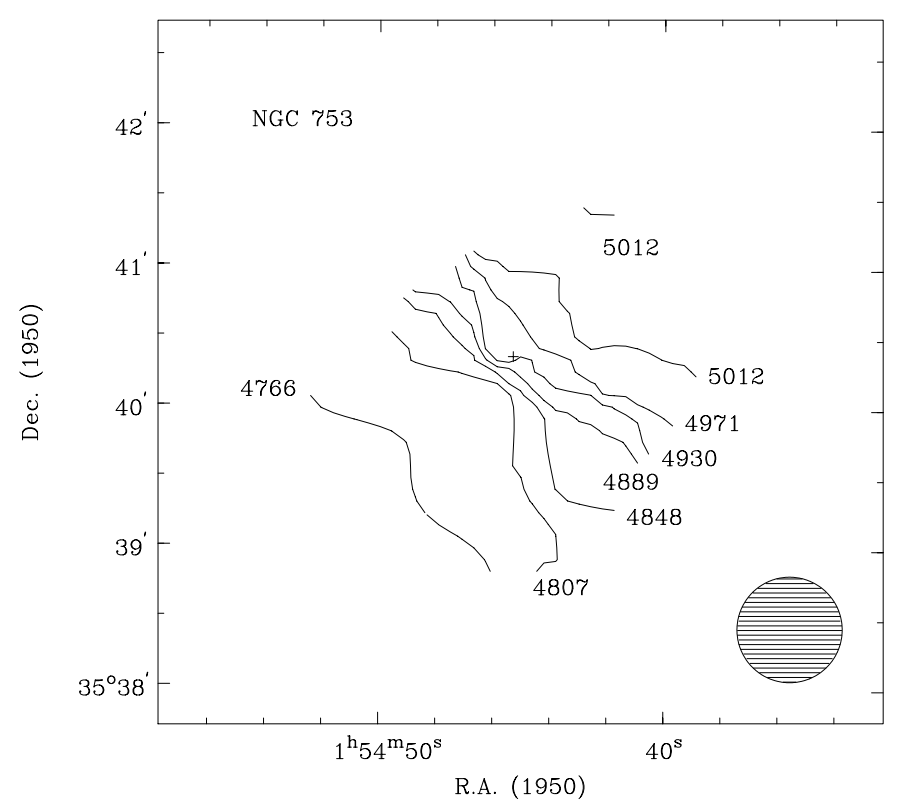

Fig. 10. b) Intensity weighted mean velocity field of NGC 753 . The numbers indicate heliocentric velocity in $\mathrm{km} \mathrm{s}^{-1}$. The FWHM is indicated by the hatched circle, $45^{\prime \prime} \times 45^{\prime \prime}$

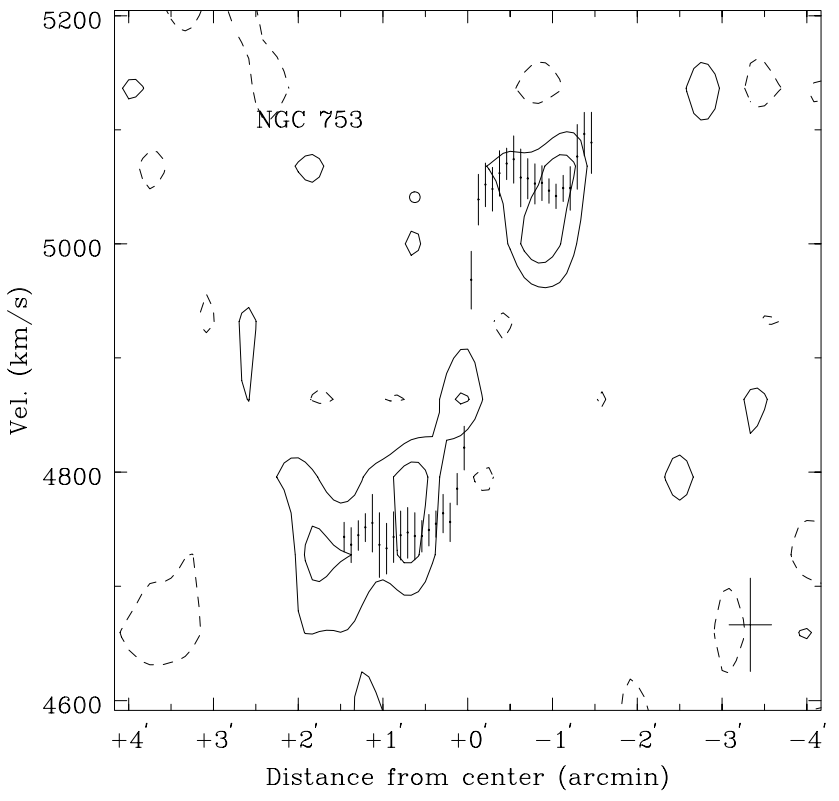

Fig. 10. c) Position velocity plot of NGC 753 along the major axis. The position angle is $128^{\circ}$. Contours are -1.3 (dashed lines), 1.3, and $2.6 \mathrm{mJy}$ beam $^{-1}$. The resolution is indicated by the cross in the lower right corner, the $\mathrm{H} \alpha$ rotation curve, from Amram et al. (1994), is plotted with the corresponding error bars 


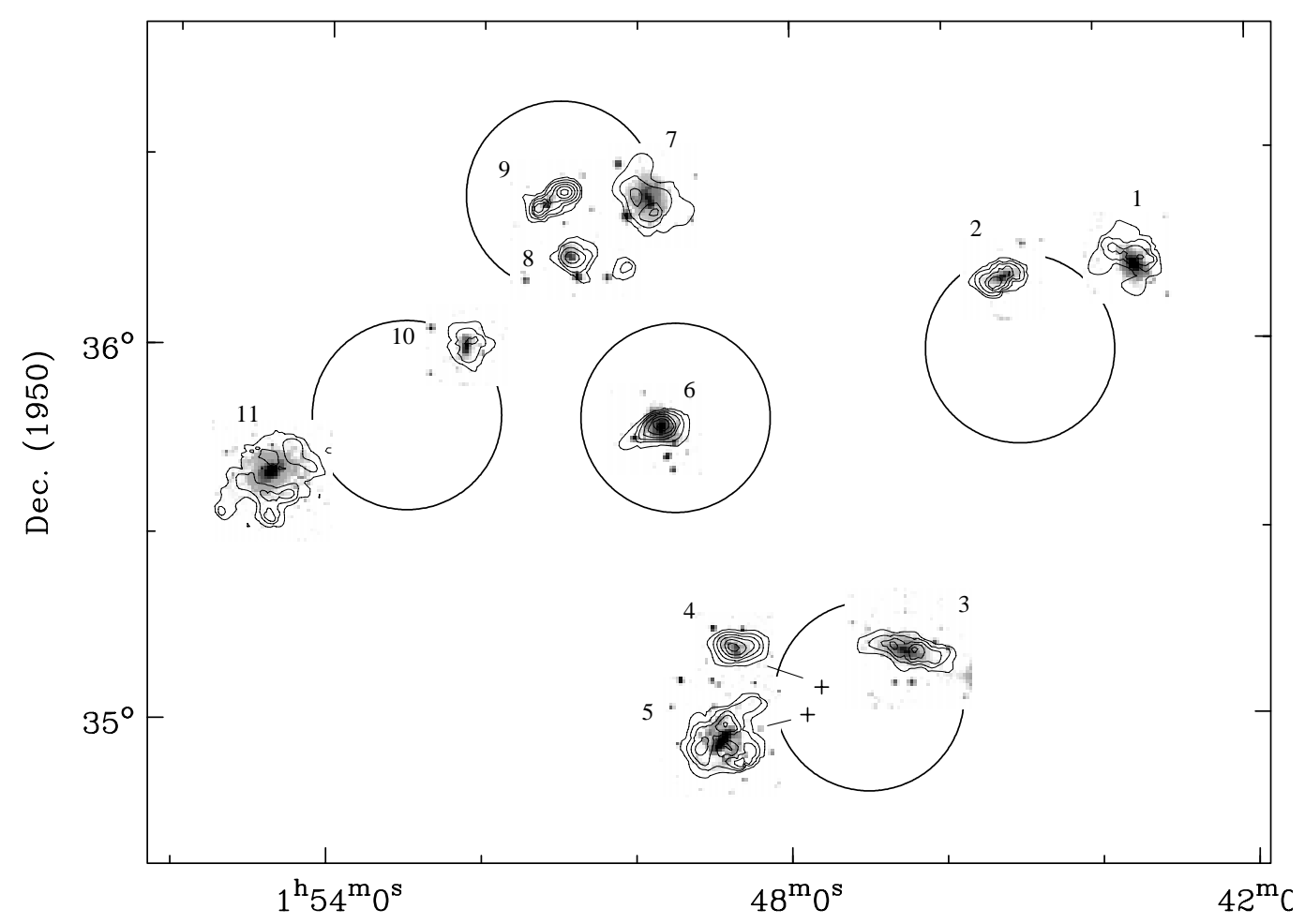

R.A. (1950)

Fig. 11. Composite plot of the integrated HI maps detected in A 262, magnified by a factor 6 . Galaxies are shown at their proper positions except when indicated by solid lines. First contours are between 1.8 and $3.910^{20} \mathrm{~cm}^{-2}$, except for NGC 668 and NGC 753 where contours begin at $4.710^{20} \mathrm{~cm}^{-2}$ and $6.410^{20} \mathrm{~cm}^{-2}$, respectively. The segmented $30^{\prime}$ diameter circles outline the 5 observed fields, and numbers list the detected galaxies in the same order as in Table 2 . The cluster center lies at $6^{\prime}$ from NGC 710 (plotted with number 6), to the North 